\title{
Analyzing of Non-Uniform MIMO Radar MMSE Beampattern Design
}

\author{
Amirsadegh Roshanzamir ${ }^{1}$, M. H. Bastani ${ }^{2}$ \\ I (Sharif university of technology) \\ ${ }^{2}$ (Sharif university of technology)
}

\begin{abstract}
Multiple Input Multiple Output (MIMO) radar is an emerging technology which has attracted many researchers recently. The problem of beampattern design of MIMO radar in uniform arrays and with the covariance based method of point targets has been investigated by many papers so far. In this paper it is desirable to consider this problem in non-uniform arrays. Actually the main purpose of this paper is to examine the optimization of the transmitted beampattern respect to transmitter antennas locations and investigated how this parameter can affect the transmitted beampattern. MATLAB software is being employed for simulations.
\end{abstract}

Keywords: - MIMO radar, non-uniform, beampattern matching design

\section{INTRODUCTION}

Multiple input multiple output (MIMO) radar is an emerging radar field which has attracted many researchers from all over the world recently. In this type of radar, unlike an standard phased array radar, one can choose transmitted probing signals freely to maximize the power around the locations of the targets of interest, or more generally to approximate a given transmit beam-pattern, or to minimize the cross-correlation of the signals reflected back to the radar by the targets of interest [1,2]. These signals can be correlated to each other or non-correlated. This feature provides extra degrees of freedom in the design of radar system.

Generally MIMO radar systems can be classified into two main categories:

MIMO radars with widely separated antennas [3]

MIMO radars with colocated antennas [4]

In the case of widely separated antennas, the transmitting antennas are far from each other such that each views a different aspect of a target of interest. In this type of MIMO radars, the concept of MIMO can be used to increase the spatial diversity of the radar system $[5,6,7,8]$. this spatial diversity which has been achieve from RCS diversity gain, can improve the performance of detection [7], finding slow moving targets [5] and angle estimation [8]. Useful references about MIMO radar with widely separated antennas can be found in [3]. In the case of MIMO radar with colocated antennas, the transmitting antennas are close to each other such that the aspects of a target of interest observed by antenna elements, are identical. In this type of MIMO radars, the concept of MIMO can be used to increase the spatial resolution. Many papers have demonstrated the advantages of this type of MIMO radars, including high interference rejection capability [9,10], improved parameter identifiability [11], and enhanced flexibility for transmit beampattern design [12,13]. Useful references about MIMO radars with colocated antennas can be found in [4].

Generally MIMO radar waveform design methods can be classified into three main categories [12] - [23]:

Covariance matrix based design methods [12] - [16]

Radar ambiguity function based design methods [17] - [20]

Extended targets based design methods [21] - [23]

In the covariance matrix based design methods, the cross correlation matrix of transmitted signals is taken into consideration instead of entire waveform. Then these types of waveform design methods affect only the spatial domain. In references $[12,14]$ the cross correlation matrix of transmitted signals is design such that the power can be transmitted to a desire range of angles. In [13] the cross correlation matrix of transmitted signals is design such that one can control the spatial power. In addition in [13] the cross correlation between the transmitted signals at a number of given target locations is minimized which can improved the spatial resolution in the radar receiver. In [15] the authors have optimized the covariance between waveforms based on the Cramer - Rao bound matrix. And finally in [16], given the optimize covariance matrix, the corresponding signal waveforms are designed to further achieve low peak to average power ratio (PAR) and higher range resolution.

The radar ambiguity based design methods optimize the entire signal instead of matrix of cross correlation of the signals. Then these design methods involve not only the spatial domain but also the range domain. Actually in these design methods it is desirable to find a set of signals which satisfied a desire radar ambiguity function. Of course these design methods are more complicated than the covariance design methods. Angle, Doppler and range resolution of a radar system can be characterized by the MIMO radar ambiguity 
function [24] - [26]. In [18] - [20] the author's goal is to sharpen the radar ambiguity function. For achieving this goal the authors have minimized the sidelobe of the autocorrelation and the cross correlation between the signals. In [17] the signals are directly optimized so that a sharper radar ambiguity function can be obtained. Then the spatial and range resolution of point targets can be improved.

In the extended target based design methods also, the entire signals is considered as in the radar ambiguity function design based methods, however, unlike the radar ambiguity function design based methods which considered only the resolutions of point targets, these methods considered the detection and estimation of extended targets. These methods require some prior information about the target or clutter impulse response. The extended target based design methods have been studied in [17] - [20].

Many papers have investigated beampattern design and waveforming in MIMO radars. One of the most famous approaches for beampattern design (as mentioned before) is named covariance based method which design cross-correlation matrix of transmitted signals instead of the signals. On the other hand, many papers have investigated beamforming of an standard phased array radar in different ways. In this paper it is desirable to design beampattern of a phased array radar by means of the MIMO radar covariance based beampattern design method, as a special case of general MIMO radar.

The present study has five sections as follow:

Section 1 presents a brief introduction of MIMO radars. In section 2 the covariance based MIMO radar beamforming is reviewed. Analyzing of transmitted beampattern respect to antenna location is discussed in section 3. Numerical examples are provided in section 4. Section 5 is focused on conclusion and references are presented at the end of the paper.

\section{COVARIANCEBASEDBEAMPATTERNDESIGN}

Assume that we have a collection of $\mathrm{N}$ transmitter antenna which is located at known coordinates $\mathrm{x}_{\mathrm{i}}=$ $\left(\mathrm{x}_{1, \mathrm{i}}, \mathrm{x}_{2, \mathrm{i}}, \mathrm{x}_{3 \mathrm{i}}\right)=(\mathrm{x}, \mathrm{y}, \mathrm{z})$ in some spherical coordinate along the $\mathrm{z}$-axis as shown in Fig. 1. In the presented study and in all of the examples and formulas of current paper, it is assumed that these transmitter antennas are along the $\mathrm{z}$-axis.

It is assume that each transmitter antenna is driven by a specific signal on the carrier frequency of $f_{c}$ or with wavelength of $\lambda$ and complex envelopeofs $s_{i}(t), i=1, \ldots, N$. At a specific point in space with distance of $r$ and direction of $\mathrm{k}(\theta, \phi)$ from the transmitter antenna, each radiated signal $\mathrm{s}_{\mathrm{i}}(\mathrm{t})$ gives rise to a "signal" the far field at radius $r$, with complex envelope given by

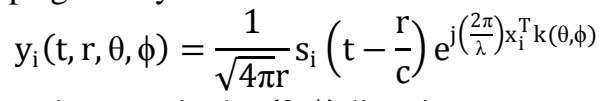

Where, in this equation, $\mathrm{k}$ is a unit vector in the $(\theta, \phi)$ direction.

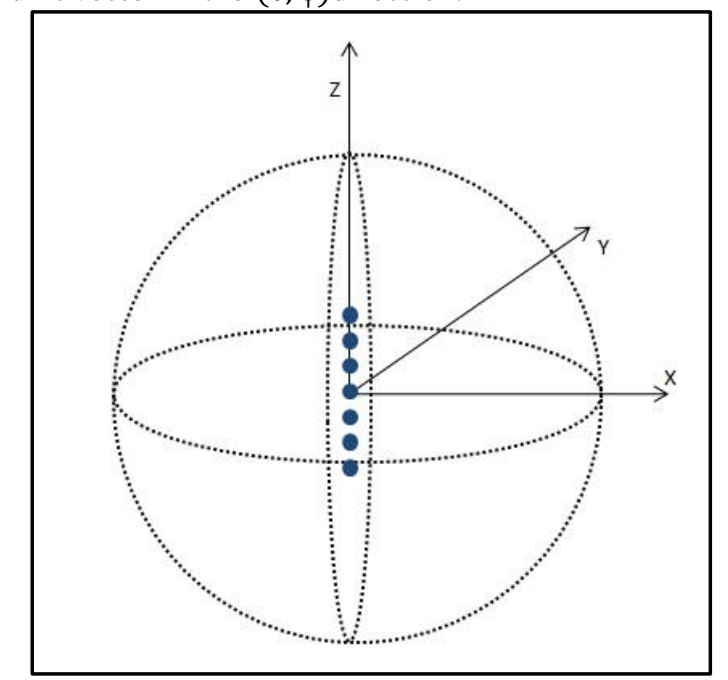

Fig. 1. T/R modules and spherical coordinate system

At the far field, these signals add linearly and the radiated powers $P_{i}$ add linearly as well. At this point assume that the $\mathrm{i}$-th element location is on the $\mathrm{z}$-axis at coordinate $z_{\mathrm{i}}$. The signal at position $(r, \theta, \phi)$ resulting from all of the transmitted signals at far field will be:

$$
y(t, r, \theta, \phi)=\sum_{i=1}^{N} y_{i}(t, r, \theta, \phi)=\frac{1}{\sqrt{4 \pi} r} \sum_{i=1}^{N} s_{i}\left(t-\frac{r}{c}\right) e^{j\left(\frac{2 \pi z_{i}}{\lambda}\right) \sin (i(\theta))}
$$

The power density of the entire signals then given by 


$$
\mathrm{P}_{\mathrm{y}}(\mathrm{r}, \theta, \phi)=\frac{1}{4 \pi \mathrm{r}^{2}} \sum_{\mathrm{k}=1}^{\mathrm{N}} \sum_{\mathrm{l}=1}^{\mathrm{N}}<\mathrm{s}_{\mathrm{k}}(\mathrm{t}) \mathrm{s}_{\mathrm{l}}^{*}(\mathrm{t})>\mathrm{e}^{\mathrm{j}\left(\frac{2 \pi\left(\mathrm{z}_{\mathrm{k}}-\mathrm{z}_{\mathrm{l}}\right)}{\lambda}\right) \sin (\theta)}
$$

And it is known that the complex signal cross-correlation is defined by

$$
\mathrm{R}_{\mathrm{kl}}=<\mathrm{s}_{\mathrm{k}}(\mathrm{t}) \mathrm{s}_{\mathrm{l}}^{*}(\mathrm{t})>
$$

With defining the direction vector as below

$$
\mathrm{a}(\theta)=\left[\mathrm{e}^{\mathrm{j}\left(\frac{2 \pi \mathrm{z}_{1}}{\lambda}\right) \sin (\theta)}, \ldots, \mathrm{e}^{\mathrm{j}\left(\frac{2 \pi \mathrm{z}_{\mathrm{N}}}{\lambda}\right) \sin (\theta)}\right]^{\mathrm{T}}
$$

The normalized power density $\mathrm{P}(\theta, \phi)$ of signals, in (W/ster), would be:

$$
\left.\mathrm{P}(\theta, \phi)=\frac{1}{4 \pi} \sum_{\mathrm{k}=1}^{\mathrm{N}} \sum_{\mathrm{l}=1}^{\mathrm{N}} \mathrm{R}_{\mathrm{kl}} \mathrm{e}^{\mathrm{j} 2 \pi} \mathrm{z}_{\mathrm{k}}-\mathrm{z}_{\mathrm{l}}\right) \sin (\theta)
$$

Recognizing that (6) is quadratic form in the Hermitian matrix $\mathrm{R}$ which is the cross-correlation matrix of signals, this can be written compactly as

$$
\mathrm{P}(\theta, \phi)=\frac{1}{4 \pi} \mathrm{a}^{*}(\theta) \operatorname{Ra}(\theta)
$$

This normalized power density $\mathrm{P}(\theta, \phi)$ is exactly the beampattern which is desirable to find[3].

In the following some examples of beampatterns produce from such a cross-correlation matrix has been shown. Fig. 2 shows the beampattern produced by signal cross-correlation matrix of (8), (9) and (10) respectively. It is noticeable that these figures are beam-patterns of 10-element uniform linear array (ULA) with half-wavelength spacing.

$\left[\begin{array}{ccc}1 & \cdots & 1 \\ \vdots & \ddots & \vdots \\ 1 & \cdots & 1\end{array}\right]$

$$
\left[\begin{array}{ccc}
0.8^{0} & \cdots & 0.8^{9} \\
\vdots & \ddots & \vdots \\
0.8^{9} & \cdots & 0.8^{0}
\end{array}\right]
$$

$\left[\begin{array}{ccc}1 & \cdots & 0 \\ \vdots & \ddots & \vdots \\ 0 & \cdots & 1\end{array}\right](10)$

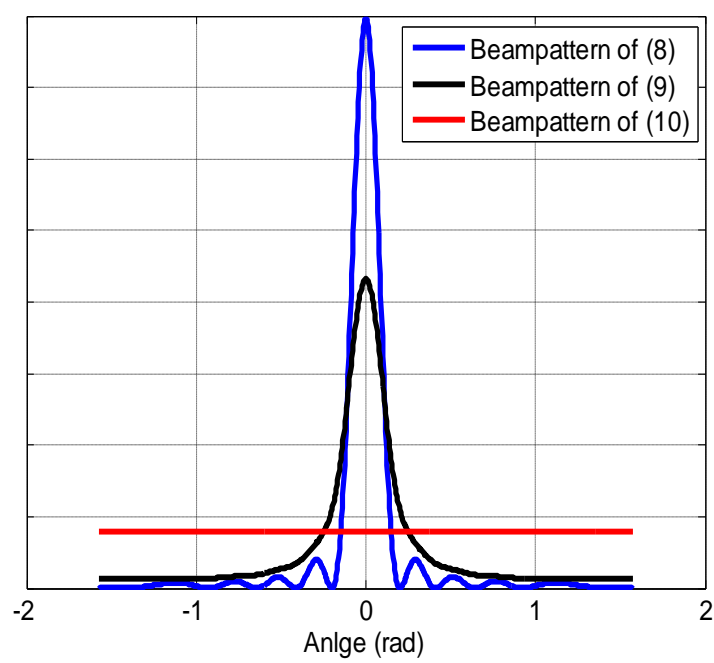

Fig. 2. Beampattern respect to (7). The blue one is corresponds to cross-correlation matrix of (8), The black one is corresponds to cross-correlation matrix of (9) and the red one is corresponds to cross-correlation matrix of

In general case the elements of the signal cross-correlation matrix are complex values except the diagonal elements that are real. This general case is related to MIMO radars but in the case of phased array radar, all the transmitter signals are correlated with each other and then absolute value of all the elements in $\mathrm{R}$, are equal to 1(blue one at Fig. 2). 


\section{ANALYZING OF TRANSMITTED BEAMP ATTERNRE SPECTTOANTENNA LOCATION}

In this section the problem of beampattern matching design is considered in the following format:

$$
J(z, R)=\int_{\theta}\left|P_{d}(\theta)-a^{*} \operatorname{Ra}(\theta)\right|^{2} d \theta
$$

Where, the goal is to minimize this function. In other words it is desirable to match the produced pattern to a desire pattern in a mean square (MS) manner. Then it is desirable to investigate how the antenna location can affect the transmitted beampattern. In other words we want to show that how an arbitrary beampattern can change by moving the transmitter antennas. As it is clear, the above problem is a $\mathrm{N}$ dimensional problem respect to antenna location, then considering the total effects of all the antennas are difficult to consider. Then in the numerical example section, the effect of change in two antennas locations is investigated. Therefore it can be shown in a plane.

\section{NUMERICALEXAMPLES}

In this section it is supposed that there is 5 elements array which two of these elements can move freely. Figure 3 shows an arbitrary desire beampattern and arbitrary transmitted beampattern which is obtained from minimum mean square error and figure 4 shows the resulting difference of transmitted beampattern and desire beampattern respect to these two moving antennas locations parameters.

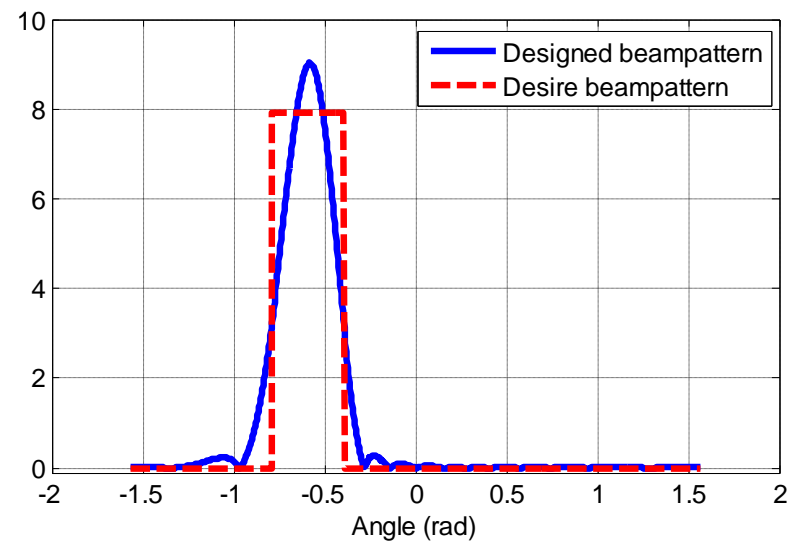

Fig. 3. Arbitrary desire and designed beampatterns

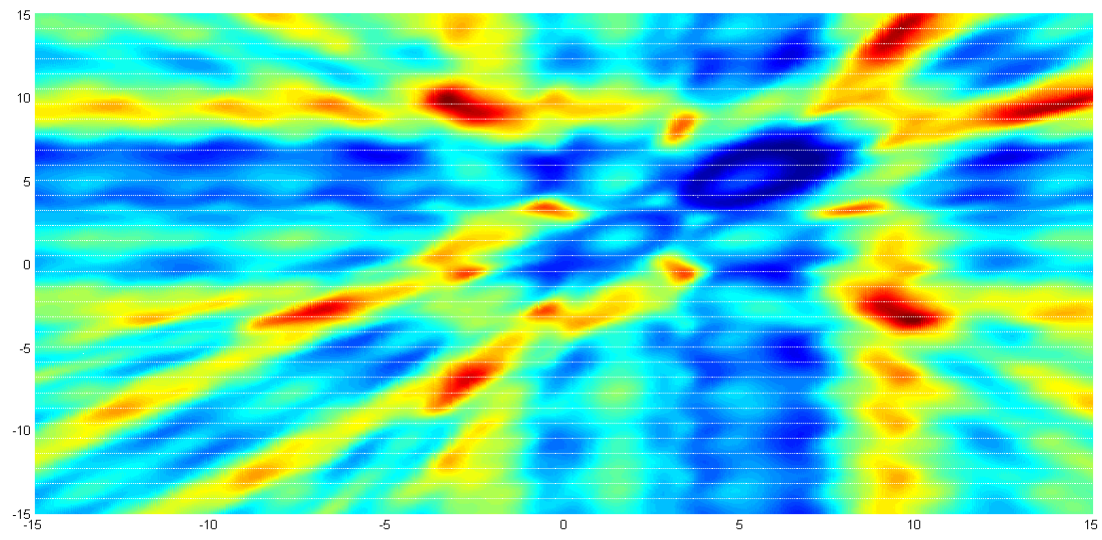

Fig. 4. The difference of transmitted beampattern and desire beampattern respect to two antennas locations

As it is seen from this figure the difference between transmitted beampattern and desire beampattern is varying in non-linear sense respect to antenna locations.

\section{CONCLUSION}

It is showed in this paper that optimizing the transmitted beampattern respect to antennas locations is an nonconvex optimization problem which cannot solve with conventional optimization methods and therefore one can solve such problems with some heuristic approaches like genetic algorithm. 


\section{REFERENCES}

[1] Chun-Yang Chen, "MIMO Radar Waveform Optimization With Prior Information of the Extended Target and Clutter" Signal Processing, IEEE Transaction Vol. 57 , Issue: 9 pp. 3533 - 3544 Sept. 2009.

[2] D. R. Fuhrmann and G. S. Antonio, "Transmit beamforming for MIMO radar systems using partial signal correlation," in Proc. 38th IEEE Asilomar Conf. Signals, Syst., Comput., Nov. 2004, pp. 295-299.

[3] D. R. Fuhrmann and G. S. Antonio, "Transmit beamforming for MIMO radar systems using signal crosscorrelation,” IEEE Trans. Aerosp. Electron. Syst., vol. 44, pp. 171-186, Jan. 2008.

[4] P. Stoica, J. Li, and Y. Xie, "On probing signal design for MIMO radar," IEEE Trans. Signal Process., vol. 55, no. 8, pp. 4151-4161, Aug. 2007.

[5] J. Li, L. Xu, P. Stoica, K. W. Forsythe, and D. Bliss, "Range compression and waveform optimization for MIMO radar: A Cramér-Rao bound based study," IEEE Trans. Signal Process., vol. 56, no. 1, pp. 218 232, Jan. 2008.

[6] P. Stoica, J. Li, and X. Zhu, "Waveform synthesis for diversity-based transmit beampattern design," IEEE Trans. Signal Process., Jun. 2008.

[7] C. Y. Chen and P. P. Vaidyanathan, "MIMO radar ambiguity properties and optimization using frequency-hopping waveforms," IEEE Trans. Signal Process., Dec. 2008.

[8] J. Li, P. Stoica, and X. Zheng, "Signal synthesis and receiver design for MIMO radar imaging," IEEE Trans. Signal Process., Aug. 2008.

[9] D. Kornack and P. Rakic, "Cell Proliferation without Neurogenesis in Adult Primate Neocortex," Science, vol. 294, Dec. 2001, pp. 2127-2130, doi:10.1126/science.1065467.

[10] B. Liu, Z. He, and Q. He, "Optimization of orthogonal discrete frequency-coding waveform based on modified genetic algorithm for MIMO radar," in Proc. Int. Conf. Commun., Circuits, Syst., Jul. 2007, pp. 966-970.

[11] B. Friedlander, "Waveform design for MIMO radars," IEEE Trans. Aerosp. Electron. Syst., vol. 43, pp. 1227-1238, Jul. 2007.

[12] Y. Yang and R. S. Blum, "MIMO radar waveform design based on mutual information and minimum mean-square error estimation," IEEE Trans. Aerosp. Electron. Syst., vol. 43, no. 1, pp. 330-343, Jan. 2007.

[13] Y. Yang and R. S. Blum, "Minimax robust MIMO radar waveform design," IEEE J. Sel. Topics Signal Process., vol. 1, no. 1, pp. 147-155, Jun. 2007.

[14] E. Fishler, A. Haimovich, R. Blum, L. Cimini, D. Chizhik, and R. Valenzuela, "Performance of MIMO radar systems: Advantages of angular diversity," in Proc. 38th Asilomar Conf. Signals, Syst. Comput., Nov. 2004, vol. 1, pp. 305-309.

[15] E. Fishler, A. Haimovich, R. Blum, D. Chizhik, L. Cimini, and R. Valenzuela, "MIMO radar: An idea whose time has come," in Proc. IEEE Radar Conf., Apr. 2004, pp. 71-78.

[16] A. M. Haimovich, R. S. Blum, and L. J. Cimini, "MIMO radar with widely separated antennas," IEEE Signal Process. Mag., vol. 25, no. 1, pp. 116-129, Jan. 2008.

[17] J. Li and P. Stoica, "MIMO radar with colocated antennas," IEEE Signal Process. Mag., vol. 24, no. 5, Sep. 2007. 